\title{
The Role of Metformin for Prevention of First Trimesteric Miscarriage in Women With Polycystic Ovary Syndrome: A Original Randomized Controlled Trial Article
}

\author{
Islam Hussein, Ahmed Abdelshafy*, Hassan Awwad \\ Ain-Shams Maternity Hospital, Faculty of Medicine, Ain-Shams University, Cairo, Egypt
}

\begin{abstract}
Background: This study aimed to evaluate the effect of metformin on early pregnancy loss (EPL) in pregnant women with olycystic ovary syndrome (PCOS).

Patients and Methods: A randomized clinical trial (Clinicaltrials.gov NCT 02498522) was conducted in Ain-Shams Maternity Hospital in the period between August 2018 and July 2019. One hundred and sixty six women previously diagnosed with PCOS and got pregnant with induction of ovulation in concomitance with Metformin were randomized either Metformin $500 \mathrm{mg}$ orally every 8 hours until the end of the $1^{\text {st }}$ trimester or not receiving Metformin. The primary outcome is to observe any significant decline in the incidence of EPL in PCOS patients after administration of Metformin through out $1^{\text {st }}$ trimester.

Results: There was a significant reduction in miscarriage rate after administration of Metformin during $1^{\text {st }}$ trimester. Introduction of Metformin helped pregnancy loss rate to drop to $10.8 \%$ while the rate in the group discontinued Metformin was $42.2 \%(p<0.05)$. The side effects rate as nausea, vomiting, gastric irritation and flatulence was significantly higher in the Metformin group.

Conclusion: The use of Metformin in patients with PCOS during $1^{\text {st }}$ trimester reduces the incidence of early pregnancy loss (EPL).
\end{abstract}

Key Words: Early pregnancy Loss (EPL), Metformin,PCOs

Received: 16 October 2019, Accepted: 16 October 2019

Corresponding Author: Ahmed Abdelshafy, Ain-Shams Maternity Hospital, Faculty of Medicine, Ain-Shams University, Cairo, Egypt,Tel.: 01069491030,E-mail: abdelshafyahmed236@gmail.com

ISSN: 2090-7625, February 2020, Vol.10, No.1

\section{INTRODUCTION}

Polycystic ovary syndrome (PCOS) is the most common endocrine disorder in women of reproductive age. PCOS is thought to be one of the leading causes of the female subfertility and produces symptoms in approximately 5 to $10 \%$ of women of reproductive age (12-45 years old $)^{[1]}$.

PCOS is a medical condition, in which there is an imbalance of the female sex hormones i.e. elevated levels of testosterone, androstenedione, prolactin, DHEA-S and LH along with a normal, high or low estrogen level and anovulation (or oligo-ovulation) in women. According to the Rotterdam criteria, at least two of which must be present to fulfill the Rotterdam criteria for the diagnosis of PCOS :

- At least twelve small follicles 2-9 $\mathrm{mm}$ in at least one ovary.

- Symptoms or biochemical evidence of hyperandrogenism.

- Anovulation or oligo-ovulation with fewer than nine menstrual periods every 12 months $^{[2]}$.
Hyperinsulinaemia, insulin resistance and impaired glucose tolerance are very common in women with PCOS, particularly in those with a body mass index $(\mathrm{BMI})>30 \mathrm{Kg} / \mathrm{m} 2^{[3]}$. Soon after the insulin resistance contribution to the pathogenesis of PCOS was recognized, it was speculated whether insulin sensitizing agents such as metformin could be useful to treat the various consequences of the condition ${ }^{[4]}$. Metformin, a biguanide, has been available for the treatment of type 2 diabetes mellitus. It has become the most widely prescribed antihyperglycaemic agent. Its mechanism of action involves the suppression of endogenous glucose production, primarily by the liver ${ }^{[5]}$. Metformin can significantly affect women of the reproductive age with PCOS, especially those who are resistant to ovulation-inducing drugs such as clomiphene ${ }^{[6]}$.

\section{AIM OF THE WORK}

The aim of this study was to evaluate the role of Metformin in reducing early pregnancy loss in pregnant patients with PCOs. 


\section{PATIENTS AND METHODS}

This randomized controlled trial was conducted in Ain-Shams Maternity Hospital, Cairo, Egypt in the period between August 2018 and July 2019 after approval of the Hospital Ethical Review Board (ERB) and registered under the NCT number: 02498522 on www.clinicaltrials.gov.

A total of 166 women with PCO received induction of ovulation in concomitance with Metformin were enrolled in our study after confirmation of pregnancy by pregnancy test or trans-vaginal ultra sound and randomized to either continuation of Metformin through out $1^{\text {st }}$ trimester or stopping Metformin.

The inclusion criteria of the study were the diagnosis of PCOS before pregnancy based on the Rotterdam criteria ; maternal age of 25 - 35 years, gestational age between 5 weeks and 13 weeks, normal serum thyroid-stimulating hormone and pregnancy with singleton fetus. The exclusion criteria were other risk factors for miscarriage such as abnormal serum karyotyping for both parents, antiphospholipid syndrome, which was excluded by anticoagulant antibodies test, uterine anomalies as excluded by transvaginal ultrasound scanning and diabetes mellitus by oral glucose tolerance test.

Early pregnancy loss was defined as spontaneous loss before 13 completed weeks of pregnancy and was documented as the absence of fetal life that was confirmed by the ultrasonography. Results were reported as means \pm standard deviation and $p<0.05$ was considered significant.

After obtaining written consents, patients were randomized to either continuing Metformin group or stopping Metformin group using a computer generated list concealed by sequentially numbers, otherwise identical, sealed envelopes (SNOSE), each containing a paper with a written code designating the assigned group.

Patients in the Metformin group received Metformin (Cedophage ${ }^{\circledR} 500 \mathrm{mg}$, CID pharma, Egypt) administered $500 \mathrm{mg}$ TID and it was used in concomitance with drugs of induction of ovulation and continued until the end of $13^{\text {th }}$ weeks . Patients on the other group discontinued Metformin used during induction of ovulation once pregnancy was diagnosed. The two groups were followed up throughout $1^{\text {st }}$ trimester.

\section{Ultrasonographic features:}

Fetal life was followed up using a Medison x5 ultrasound machine with 4.0 MHZ trans-abdominal probe at the sonography unit at Ain-Shams University Maternity Hospital by the same experienced sonographer weekly.

\section{Sample Justification:}

The required sample size has been calculated using the G*Power Software (Universität Düsseldorf, Germany). The primary outcome measure was the incidence of spontaneous miscarriage.

Since there is currently no adequate information regarding the expected difference in the outcome measure about the suggested preventive regimens under investigation, the current exploratory study targeted an effect size that would be clinically relevant. So, it was estimated that a total sample size of 166 patients equally randomized into either study group ( $\mathrm{n}=83$ patients per group) would achieve a power of $90 \%$ (type II error, 0.1) to detect a statistically significant difference between the two groups as regards the occurrence of spontaneous miscarriage for a medium effect size (w) of 0.3 using a two-sided chi-squared test with a confidence level of $99 \%$ (type I error, 0.01). The effect size (w) is calculated as follows: $\mathrm{w}=\sqrt{ } \chi^{2} / \mathrm{N}$, where $\chi^{2}$ is the chi-squared statistic and $\mathrm{N}$ is the total sample size.

An effect size of $\mathrm{w}=0.3$ for the difference in the outcome measure has been chosen as it could be regarded as clinically relevant difference to seek in this exploratory study.

\section{STATISTICAL ANALYSIS:}

Data was analyzed using IBM( SPSS $\odot$ Statistics version 22 (IBM(C) Corp., Armonk, NY). Normally distributed numerical data were presented as mean and SD, and skewed data as median and interquartile range. Qualitative data were numbered and percentage. Comparison of normally distributed numerical data was done using the unpaired t test. Skewed data were compared using the Mann-Whitney test. Categorical data were compared using the Pearson chi-squared test or fisher's exact test, if appropriate. A two-sided $p$-value $<0.05$ was considered statistically significant.

\section{RESULTS}

One hundred and sixty-six women with PCOS which was diagnosed by the Rotterdam criteria who received induction of ovulation in concomitance with Metformin and got pregnant were randomly assigned to either continuation of Metformin throughout 1st trimester $(\mathrm{n}=83$ ) or stopping Metformin as soon as pregnancy was diagnosed $(n=83)$. The process of recruitment and handling of the study population during the course of the study is shown in the CONSORT flow diagram (Figure 1).

No statistically significant differences were found 
between both groups regarding basal demographic and clinical characteristics (Table 1).

Early pregnancy Loss (EPL) rate was significantly lower in the Metformin group compared to the group stopped Metformin (Table 2).
As shown in (Table 3) body mass index (BMI) was significantly high in both groups.

There was a significantly higher rate of gastrointestinal side effects in Metformin group and exaggeration of pregnancy symptoms in the Metformin group (Figure 2).

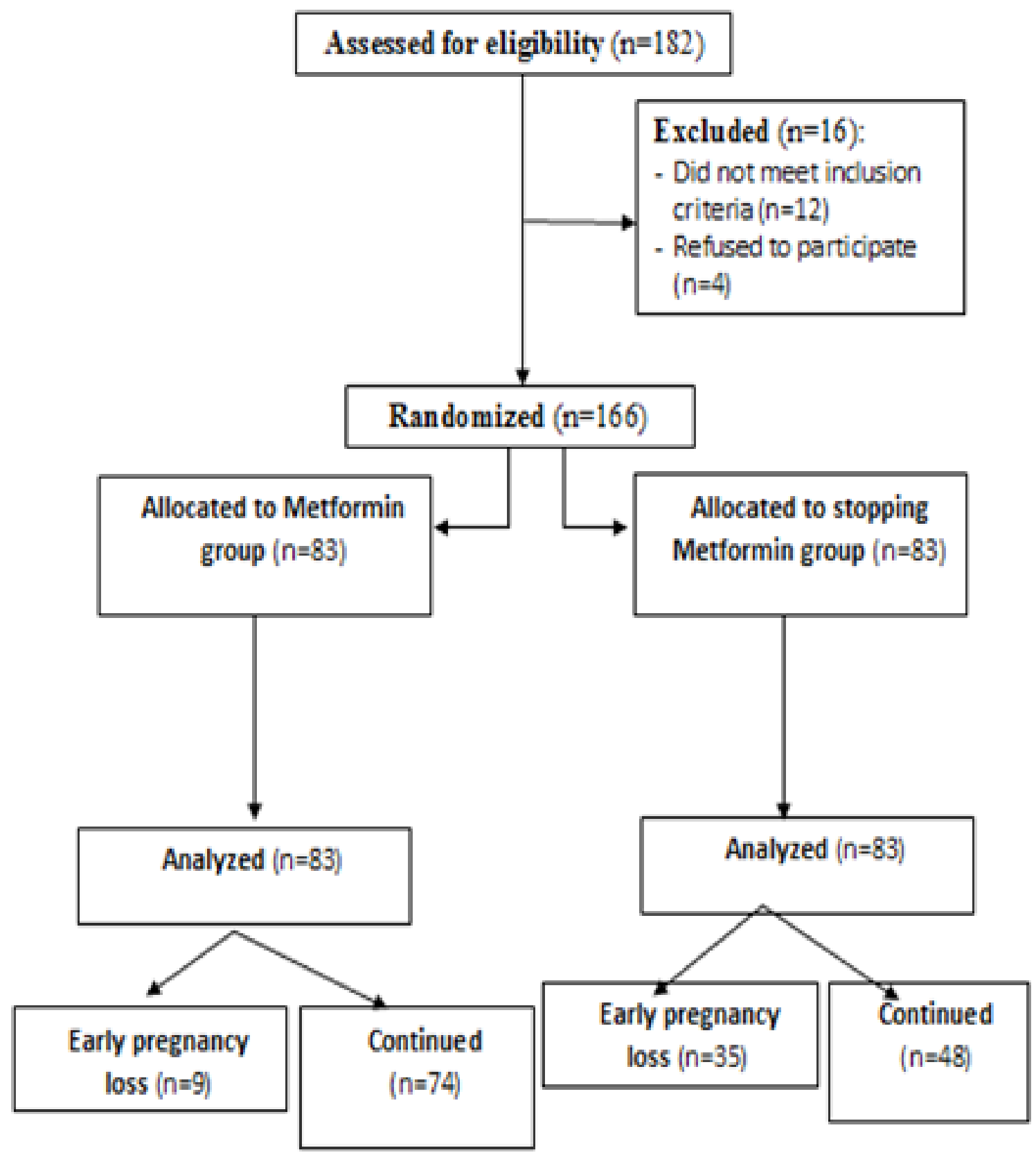

Fig. 1 : CONSORT flow diagram showing the recruitment and handling of the study population during course of the study 


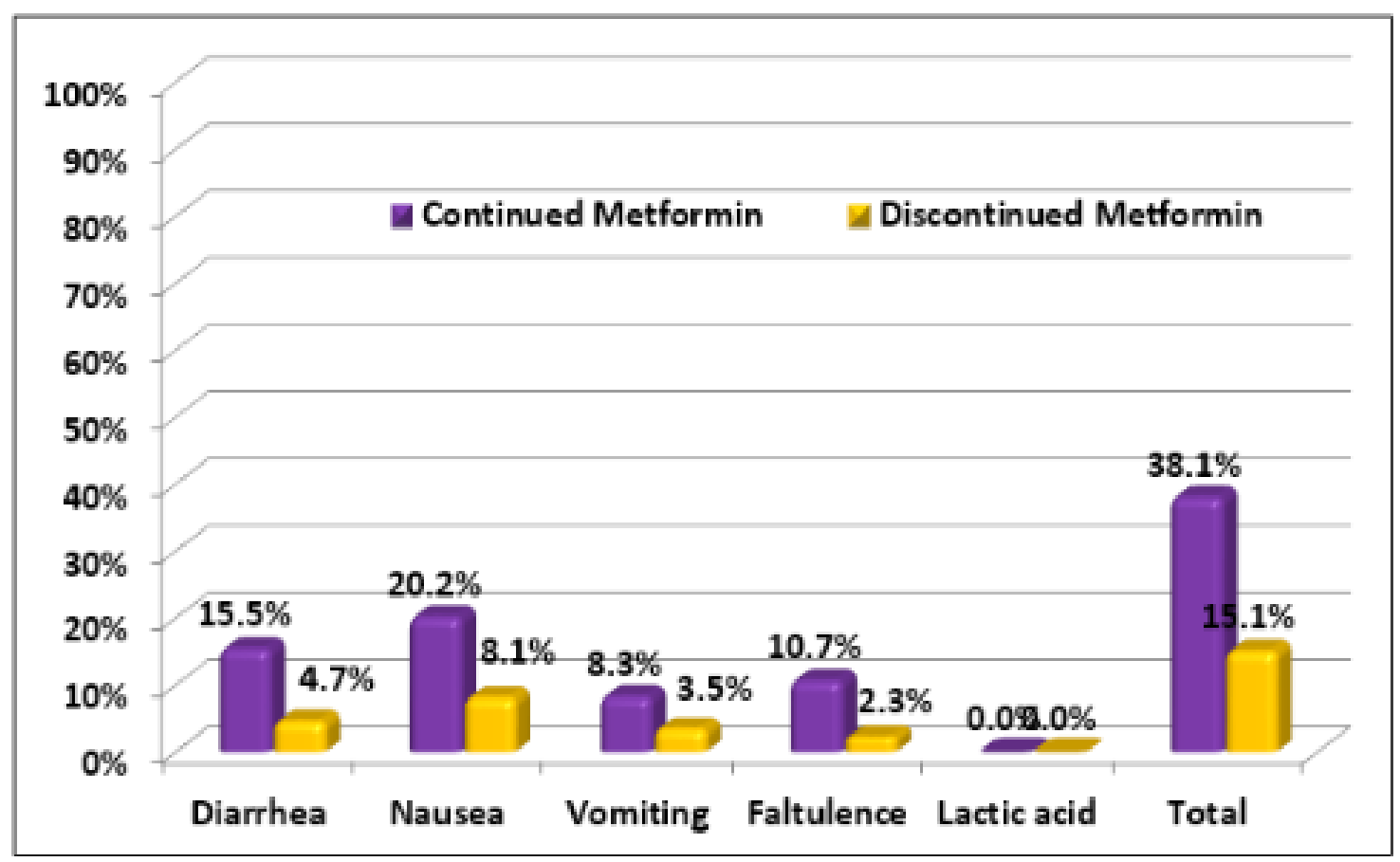

Fig. 2: Prevalence of side effects in study groups

Table 1: Basal demographic characteristics in the two study groups

\begin{tabular}{|c|c|c|c|c|}
\hline & & Study $(\mathrm{N}=38)$ & Control (N-38) & $\wedge P$ \\
\hline \multirow[t]{3}{*}{ Age (Years) } & Mean \pm SD & $30.2 \pm 1.6$ & $30.1 \pm 1.9$ & \multirow{3}{*}{0.724} \\
\hline & & & & \\
\hline & Range & $26.0 \pm 35.0$ & $25.0 \pm 35.0$ & \\
\hline \multirow[t]{3}{*}{ BMI $\left(\mathrm{Kg} / \mathrm{m}^{2}\right)$} & Mean \pm SD & $28.8 \pm 1.5$ & $28.8 \pm 1.4$ & \multirow{3}{*}{0.588} \\
\hline & & & & \\
\hline & Range & $25.3 \pm 31.9$ & $25.4-32.7$ & \\
\hline \multirow[t]{3}{*}{ Parity } & Mean \pm SD & $2.0 \pm 0.9$ & $2.0 \pm 0.8$ & \multirow{3}{*}{0.649} \\
\hline & & & & \\
\hline & Range & $0.0-4.0$ & $0.0-4.0$ & \\
\hline \multirow{2}{*}{$\begin{array}{l}\text { Previous early } \\
\text { pregnancy loss }\end{array}$} & Mean \pm SD & $1.5 \pm 0.5$ & $1.6 \pm 0.6$ & \multirow{2}{*}{0.511} \\
\hline & Range & $1.0-3.0$ & $1.0-3.0$ & \\
\hline
\end{tabular}

${ }^{\wedge}$ Independent $\mathrm{t}$-test 
Table 2: EPL among the study groups

\begin{tabular}{|c|c|c|c|}
\hline Measures & Study $(\mathrm{N}=38)$ & Control $(\mathrm{N}=38)$ & $\# p$ \\
\hline $\mathrm{n}, \%$ & $9(10.8 \%)$ & $35(42.2 \%)$ & $0.005 *$ \\
\hline $95 \% \mathrm{CI}$ & $4.1 \%-17.5 \%$ & $31.6 \%-52.8 \%$ & \\
\hline \multicolumn{4}{|c|}{ Efficacy of continuing metformin in preventing early pregnancy loss } \\
\hline & Items & Value & $95 \% \mathrm{CI}$ \\
\hline Rate in study group & & $89.2 \%$ & $82.5 \%-95.9 \%$ \\
\hline Rate in control group & & $57.8 \%$ & $47.2 \%-68.4 \%$ \\
\hline Rate elevation & & $31.3 \%$ & $17.3 \%-41.6 \%$ \\
\hline Relative rate & & 1.542 & $1.266-1.789$ \\
\hline Number needed to prevent & & 3.2 & $2.4-5.8$ \\
\hline
\end{tabular}

\#Chi square test, *Significant, CI:confidence interval

Table 3: Effect of BMI on EPL

\begin{tabular}{|c|c|c|c|c|}
\hline & & EPL & Continued & $\wedge P$ \\
\hline \multirow[t]{2}{*}{ Age (Years) } & Study & $30.1 \pm 0.9$ & $30.2 \pm 1.7$ & 0.929 \\
\hline & Control & $29.9 \pm 1.8$ & $30.1 \pm 2.0$ & 0.631 \\
\hline \multirow[t]{2}{*}{ BMI $\left(\mathrm{Kg} / \mathrm{m}^{2}\right)$} & Study & $30.0 \pm 0.9$ & $28.7 \pm 1.5$ & $0.014 *$ \\
\hline & Control & $29.3 \pm 1.2$ & $28.6 \pm 1.5$ & $0.025^{*}$ \\
\hline \multirow[t]{2}{*}{ Parity } & Study & $1.9 \pm 0.3$ & $2.0 \pm 0.9$ & 0.635 \\
\hline & Control & $1.9 \pm 0.8$ & $2.0 \pm 0.8$ & 0.452 \\
\hline \multirow{2}{*}{$\begin{array}{l}\text { Previous early } \\
\text { pregnancy loss }\end{array}$} & Study & $1.7 \pm 0.5$ & $1.5 \pm 0.5$ & 0.373 \\
\hline & Control & $1.5 \pm 0.7$ & $1.6 \pm 0.6$ & 0.672 \\
\hline
\end{tabular}

${ }^{\wedge}$ Independent t-test, *Significant 


\section{DISCUSSION}

Women with insulin resistance are at increased risk of hyperinsulinemia, PCOS and hyperandrogenism. They are also at risk of reduced fertility due to ovulatory dysfunction and suboptimal hormonal profiles that may impair conception and implantation.

This emphasizes the need for a treatment using drugs such as metformin, which will actively reduce insulin resistance and will restore ovulatory cycles and reduce early pregnancy loss. In addition to this, there is accumulating evidence suggesting that this drug is probably safe in the first trimester of pregnancy despite the traditional response that all oral hypoglycemic agents are contraindicated in pregnancy.

We found in our study that, there was a significant reduction in miscarriage rate after administration of Metformin during $1^{\text {st }}$ trimester. Introduction of Metformin helped pregnancy loss rate to drop to $10.8 \%$; while the rate in the group discontinued Metformin was $42.2 \%(p<0.05)$. The side effects rate as nausea, vomiting, gastric irritation and flatulence was significantly higher in the Metformin group $38.1 \%$ compared to $15.1 \%$ in the other group but with no serious side effects (i.e.: lactic acidosis).

These results are similar to those of a pilot study by Glueck C.J where twenty-two previously oligoamenorrheic, nondiabetic women with PCOS, who were not currently pregnant and who had 1 or more previous pregnancy while they were not receiving metformin.

Metformin, 1.5-2.5 g/day, throughout pregnancy was administered to monitor the rates of first trimester miscarriage and teratogenicity of metformin.

Before metformin, 10 women had 22 previous pregnancies with 16 first-trimester spontaneous abortions $(73 \%)$; on receiving metformin 1 of $10(10 \%)$ had first-trimester spontaneous abortion compared with $73 \%$ in 22 previous pregnancies without metformin $(P<.002)$.

Sonography showed normal fetal development without congenital defects in the ongoing pregnancies $^{[6]}$.

Another study conducted by Sohrabvand F where a total of 75 pregnant women with PCOS were studied in three different groups. In Group A, metformin administration [500 $\mathrm{mg}$ three times daily (TDS)] was stopped immediately after diagnosis of pregnancy (5-6 weeks gestation) While in Group B, metformin was administered until the end of 8 weeks gestation and in Group C until the end of 12 weeks gestation. The results of this study were then assessed using chi-square McNemar's, ANOVA Kruskal Wallis and logistic regression tests.

There was a significant statistical difference between previous and current miscarriage in the current pregnancy with a decline in Group B from $40 \%$ to $8 \%$ and in group C from $32 \%$ to $4 \%$. In spite of the reduced rate of miscarriage seen in Group A, from $20 \%$ to $4 \%$, this difference was not statistically significant due to small sample size ${ }^{[7]}$.

Although the mean BMI was not significantly different, high BMI was associated with a higher rate of early pregnancy loss.

These observations may be explained by the adverse effect of high insulin resistance, which is more prominent with high $\mathrm{BMI}^{[8]}$.

The current study revealed similar results regarding primary outcome (effect of metformin on decreasing EPL) as well as secondary outcome (relation between BMI and EPL) where a negative correlation was observed.

On the contrary, a double blinded multi-center randomized trial was undertaken by N.P. Johnson including 171 women with anovulatory or oligoovulatory polycystic ovary syndrome. Women with high body mass index (BMI) of $32 \mathrm{~kg} / \mathrm{m} 2$ or more received placebo ('standard care') or metformin; women with $\mathrm{BMI} \leq 32 \mathrm{~kg} / \mathrm{m} 2$ received $\mathrm{CC}$ ('standard care'), metformin or both. Treatment continued for 6 months or until pregnancy was confirmed. Primary outcomes were clinical pregnancy and live birth and a secondary outcome of the study was the rate of EPL in both groups.

Results showed that there is no evidence that adding metformin to standard care is beneficial. Pregnancy and live birth rates are low in women with $\mathrm{BMI} \geq 32$ $\mathrm{kg} / \mathrm{m} 2$ whatever treatment is used, with no evidence of benefit of metformin over placebo ${ }^{[9]}$.

We reported in our results that side effects as nausea, vomiting, diarrhea and flatulence were significantly higher in Metformin group compared to the non Metformin group. Our results are matching with Laure Morin-Papunen who found that the main side effects of Metformin were gastric upset symptoms and signs but were mild and tolerable by patients ${ }^{[10]}$.

The main strength of this study is that it was a 
randomized control trial with low risk of bias; however it was limited by not assessing the long term effect of Metformin on $2^{\text {nd }}$ and $3^{\text {rd }}$ trimesters and neonatal morbidity and mortality. It is hopeful that future trials would be designed to assess those outcomes.

\section{CONCLUSION}

In conclusion, the continued use of metformin in pregnant women with PCOS during 1 st trimester was associated with a significant reduction in the rates of early pregnancy loss. It was well tolerated by patients with a minimum of side effects. However, extended studies are required to evaluate its effect on further pregnancy complications and fetal outcomes.

\section{CONFLICT OF INTEREST}

There are no conflict of interests.

\section{REFERENCES}

1. Goldenberg N, Glueck C. (2008) Medical therapy in women with polycystic ovarian syndrome before and during pregnancy and lactation. Minerva Ginecol. 60:63-75.

2. Rotterdam ESHRE/ASRM-Sponsored PCOS consensus workshop group.(2004) Revised 2003 consensus on diagnostic criteria and long-term health risks related to polycystic ovary syndrome (PCOS). Hum Reprod 19:41-7.

3. Ehrmann DA, Cavaghan MK, Barnes RB, Rosenfield RL, Imperial J.(1999) Prevalence of impaired glucose tolerance and diabetes in women with polycystic ovary syndrome. Diabetes Care. 22:141-6.
4. Al-Inany H, Johnson N.(2006) Drugs for anovulatory infertility in polycystic ovary syndrome. BMJ 332:1461-2.

5. Hundal RS, Inzucchi SE (2003) Metformin: new understandings, new uses. 63(18):1879-94.

6. Glueck CJ, Phillips H, Cameron D, et al.(2001) Continuing metformin throughout pregnancy in women with polycystic ovary syndrome appears to safely reduce first-trimester spontaneous abortion: a pilot study. Fertil Steril 75:46-52.

7. Sohrabvand F, Shariat M, Haghollahi F, Bagheri B.(2009) Effect of metformin on miscarriage in pregnant patients with polycystic overy syndrome. West Indian Med J. 58(5) : 433-436

8. Fedorcsak $\mathrm{P}$, Storeng $\mathrm{R}$, Dale PO, Tanbo $\mathrm{T}$, Abyholm T (2000): Obesity is a risk factor for early pregnancy loss after IVF or ICSI. Acta Obstet Gynecol Scand 79: 43e8.

9. Johnson NP, Stewart AW, Falkiner J, Farquhar CM, et al.(2010) PCOSMIC : a multi-center randomized trial in women with Polycyctic Ovary Syndrome evaluating Metformin for infertility with Clomiphiene. Human Reproduction 25 (7), 1675-1683

10. Laure Morin-Papunen, Anni S. Rantala, Leila Unkila-Kallio, Aila Tiitinen, et al.(2012). Metformin Improves Pregnancy and Live-Birth Rates in Women with Polycystic Ovary Syndrome (PCOS): A Multicenter, Double-Blind, PlaceboControlled Randomized Trial. J Clin Endocrinol Metab, 97(5):1492-1500 